http://jmscr.igmpublication.org/home/ ISSN (e)-2347-176x ISSN (p) 2455-0450

crossref DOI: https://dx.doi.org/10.18535/jmscr/v9i3.21

\title{
Clinical and Diagnostic Profile of Pediatric COVID-19 Patients Admitted In Silchar Medical College and Hospital
}

Authors

\author{
Dr Sumit Das ${ }^{1}$, Dr Subhurendu Shekhar Sen ${ }^{2}$, Dr Pranav N Saji ${ }^{3}$ \\ ${ }^{1}$ Asso. Professor, Department of Pediatrics, Silchar Medical College, Silchar \\ ${ }^{2}$ Asso. Professor, Department of Microbiology, Silchar Medical College, Silchar \\ ${ }^{3}$ PGT, Department of Pediatrics, Silchar Medical College, Silchar \\ *Corresponding Author \\ Dr Anupama Deka
}

Professor and HOD, Department of Pediatrics, Silchar Medical College, Silchar

\begin{abstract}
Introduction: Though there have been a growing number of studies focused on COVID-19, limited data is available on epidemiological features, clinical manifestations, and transmission patterns in children with COVID-19, more so from India

Methods: Retrospective study regarding the clinical and laboratory profile of pediatric COVID patients and their outcomes were studied presenting between March 2020 and August 2020 with positive nasopharyngeal swab for SARS-CoV-2 by Rapid antigen testing or RT-PCR

Results: 50 COVID positive cases included in the study. Out of which 18 where neonates. Among neonates 10 out of 18 positive cases had preterm birth. Complication noted in these babies are Neonatal jaundice in 4 babies, birth asphyxia 2 cases, Respiratory distress syndrome in 3 case and one baby had meningitis. Metabolic complication like hypocalcemia and hypoglycemia noted in 2 babies. CRP found to be elevated for 5 [27.7\%] babies. Among the 32COVID positive children admitted in the COVID ward, 15 did not have any complaints. Among the 17 symptomatic children ten were having fever as one of the main complaint. Upper respiratory infection in 6 patients and 3patient had pneumonia, 5 patients presented with vomiting, loose stool or both, 1 had simple febrile convulsion, 1 patient presented with AGN. Leukocytosis was seen in 15 (46\%) children. Lymphopenia4 patients [12.5\%], CRP 8 [25\%] and elevated ESR 6[18.5\%] are noted in the pediatric COVID cases. No mortality occurred in the study group.
\end{abstract}

Conclusion: Children at all ages are prone to COVID-19 and morbidity and mortality of COVID positive cases are less when compared to adult patients.

Keywords: SARS-CoV-2, Symptoms, Laboratory profile, Outcome.

\section{Introduction}

Severe acute respiratory syndrome coronavirus 2 detected as the causative organism for the coronavirus outbreak in Wuhan in 2019. The
World Health organization has named it coronavirus disease 2019 (COVID-19) and it has caused a Public Health Emergency of International Importance ${ }^{[1-3]}$. This epidemic spread 
so fast, started in China and then to other countries in such a way that the World Health Organization proclaimed COVID-19 a pandemic on March 11, 2020 $0^{[4,5]}$. Fever, cough, dyspnea, and pneumonia are typical symptoms of adults afflicted with SARS-CoV-2. Elderly people who have underlying disorders or are immunocompromised are more likely to have major problems like acute respiratory distress syndrome $^{[6,7]}$. It's unclear why there are very few pediatric cases even though the children's immune systems are still evolving and therefore may be more vulnerable to the virus. However number of cases in children, on the other hand, has been gradually growing as the virus spreads ${ }^{[8]}$.

The clinical presentation in paediatric cases has been less severe than in adult cases, and the reason for this discrepancy is unclear ${ }^{[9]}$. Pregnant women were also advised to remain indoors since the virus's long- and short-term effects on the foetus, as well as the risk of mother-to-child vertical transmission, are unclear. Fever and cough are the most frequent clinical symptoms, according to a meta-analysis released in early $\operatorname{March}^{[10]}$. The most prominent laboratory anomalies are hypoalbuminemia, lymphopenia, and elevated inflammatory markers such as Creactive protein, lactate dehydrogenase (LDH), and erythrocyte sedimentation rate (ESR). In terms of imaging results, two-thirds of patients' chest X-rays showed ground-glass opacity (GGO). Intensive care needed in at least $20 \%$ of patients with COVID-19, especially those with comorbidities. However, Centers for Disease Control and Prevention (CDC) reported a multisystem inflammatory syndrome in children (MIS-C) associated with COVID-19 ${ }^{[11]}$. This is seen in pediatric patients manifesting with severe inflammation, multi-organ failure, and testing positive for SARS-COVID $2^{[12,13]}$

Despite the fact that serious or critical disease has been reported, and many deaths have resulted, children are likely to have milder effects and a better prognosis. As such, the following are our concerns: we ask the following questions: What are the characteristics of children with COVID-19 in terms of demographics, clinical, laboratory, and imaging? What will be the outcome in children? Through this research, we hope to find answers to these questions. There are few studies in India on the clinical and diagnostic profile of COVID 19 patients, especially among pediatric age group. This retrospective study which done in our center presents the demographic, clinical characteristics, treatment and outcome care of neonates and children with SARS-CoV-2 positivity.

\section{Method}

The present study, which was a retrospective study, conducted at Silchar medical college and hospital, a tertiary care hospital in Assam, India. Medical record review of all children admitted to the hospital between March 2020 and August 2020 were collected retrospectively. All children with either reverse transcriptase-polymerase chain reaction (RT-PCR) or Rapid Antigen Test (RAT) positive for SARS-CoV-2 were studied. Children those who tested positive were admitted to the isolation ward, which was specially created as per national guidelines. Historical details and preexisting co-morbidities were recorded.

The enrolled children were divided into two groups. Those who are in the neonatal age group and those in pediatric age group other than neonates. Detailed antenatal history along with the pregnancy complications developed during the antenatal period is collected along with detailed natal history is collected. All the neonates admitted in COVID NICU examined twice a day and the complications developed was noted. Detailed history regarding the presenting complaints and the complication developed were collected along with the blood investigations. All the patients admitted were examined twice a day.

Baseline laboratory parameters (complete hemogram and C-reactive protein) were evaluated and repeated as required. Chest radiograph was done in all symptomatic children. Suspected cases of COVID-19 without positive RT-PCR/ Rapid Antigen Test were excluded from this study. Our 
outcomes of interest were to describe the clinical signs, imaging findings, and laboratory results characteristic of pediatric patients with confirmed COVID-19.

\section{Statistical analysis}

Statistical methods (descriptive studies, chi square test, independent samples t-test) were carried out through the SPSS for windows version 16.0 .The $\mathrm{p}$ value value $<0.05$ is taken as significant.

\section{Results}

\section{Neonates}

188 antenatal mothers tested positive for SARS CoV2 infection. Eighteen neonates (9.5\%) tested RTPCR positive for SARS-CoV-2 infection. It is also seen that preterm birth is more common in babies whose mothers were tested COVID positive. 10 out of 18 positive cases had preterm birth, 8 had term delivery. All the neonates without any complication were roomed-in with their mothers in the COVID postnatal wards. None of the cases were RAT positive. Everyone was tested RTPCR positive. Complication noted for these babies are Neonatal jaundice in 4 babies, birth asphyxia 2 cases, Respiratory distress syndrome in 3 caseand one baby had meningitis. Metabolic complication like hypocalcemia and hypoglycemia noted for 2 babies. CRP found to be elevated for 5 [27.7\%] babies. No mortality occurred in neonatal age group.

\section{Pediatric Age Group}

Among the 32COVID positive children admitted in the COVID ward, 15did not have any complaints and 17[53.1\%] had some symptoms. $54 \%$ of the individuals were male and $46 \%$ were female. Mean age group of pediatric COVID patients were 6.2 years and $76 \%$ of patients were exposed to a family member who was diagnosed with COVID-19. Among the 17 symptomatic children ten were having fever as one of the main complaint. Upper respiratory infection in the form of cough and or rhinorrhea is noticed in 6 patients and 3 patients were diagnosed to have pneumonia. All those who developed pneumonia were below 5 years and none of them developed severe pneumonia, 5 patients presented with vomiting, loose stool or both these symptoms, 1 had simple febrile convulsion, 1 patient presented with AGN with congestive cardiac failure. One patient was a diagnosed case of ALL and was on chemotherapy. Among children which were symptomatic it is seen that majority of them are not immunized up to the age and is statistically significant (p-value: $<0.05)$ when compared to children which are symptomatic. 12 out of 17 which developed some symptoms did not have BCG scar. Leukocytosis was seen in 15 (46\%) children. Lymphopenia seen in 4 patients and thrombocytopenia was not seen in any child. CRP was found to be elevated for 8 [25\%] of COVID positive cases and elevated CRP is seen mainly in moderate to severe disease and is found to be statistically not significant ( $\mathrm{p}$-value :>0.05) when compared to mild COVID positive cases. ESR is found to be elevated in 6 patients and is also found that higher ESR is found mainly in COVID positive cases with moderate to severe disease and is found to be statistically significant (p-value : <0.05) when compared to mild COVID positive cases. Chest radiograph was done in all symptomatic patient and it was found to be normal in 15 (88.2\%), while two showed bilateral lower zone haziness.

Table 1: Age and sex wise distribution, clinical symptoms and disease severity of COVID-19 confirmed pediatric patients.

\begin{tabular}{|l|c|}
\hline Agewise distribution & 9 \\
\hline 1 month- 1year & 9 \\
\hline 1-5 Year & 10 \\
\hline 5-10year & 4 \\
\hline >10 year \\
\hline Sex Distribution \\
\hline Male:Female \\
\hline Symptoms at presentation \\
\hline Asymptomatic & $54: 46$ \\
\hline Fever & 15 \\
\hline Upper respiratory & 10 \\
\hline Lower respiratory & 6 \\
\hline Gastrointestinal & 2 \\
\hline Seizure & 5 \\
\hline AGN & 1 \\
\hline Disease severity & 1 \\
\hline Mild & 25 \\
\hline Moderate & 5 \\
\hline Severe & 2 \\
\hline
\end{tabular}




\section{Discussion}

Our knowledge on COVID 19 patients and its epidemiologic and clinical characteristics continue to evolve over the last one year. Most of the studies were focused on symptomatic adults. Studies regarding the COVID 19 in pediatric population is limited and it is important for the identification of clinical and laboratory characteristics in the pediatric population is essential to guide clinical care, predict disease severity, and determine prognosis. Ansel Hoanga and Kevin Chorath ${ }^{14}$ study showed fever $(59.1 \%)$ and cough $(55.9 \%)$ as the most frequent symptoms and $19.3 \%$ of children were asymptomatic. Our study showed that $17[53.1 \%]$ had some symptoms, fever 10[31.2\%] and cough $8[25 \%]$ as the more common symptom in our study and is comparatively less in our study. In the present study 15 [46.9\%] were asymptomatic compared to the study done by Ansel Hoanga and Kevin Chorath ${ }^{15}$ which showed $19.3 \%$ of children were asymptomatic. 47 (38\%) had co-morbidities in the study conducted by Sudha Rao ${ }^{15}$ while in the present study only one patient showed associated comorbidity which is much lesser compared to the other study. Bhakti Sarangi ${ }^{16}$ study showed $29(58 \%)$ children as asymptomatic COVID positive and $20(40 \%)$ had mild symptoms. Fever, cough, and sore throat were the most common symptoms, which is almost comparable with our study. High C-reactive protein levels noticed in $15(30 \%)$ children in the study conducted by Bhakti Sarangi ${ }^{16}$ while present study in pediatric age group showed elevated CRP in $8[25 \%$ ] patients, which is similar to our present study. None of the patients developes severe COVID/ MISC. No mortality was there in our study and the study done by Bhakti Sarangi ${ }^{16}$ shows no mortality whiles the study by Sudha Rao ${ }^{15}$ shows 14 (11.4\%) deaths.

\section{Conclusions}

During the study period 50 children including neonates admitted in pediatric COVID ward due to COVID 19 infection. Few of them were symptomatic. None of the patients developed severe COVID/ MISC and there was no mortality/ post COVID morbidity.

\section{References}

1. N. Chen, M. Zhou, X. Dong, et al., Epidemiological and clinical characteristics of 99 cases of 2019 novel coronavirus pneumonia in Wuhan, China: a descriptive study [J], Lancet 395 (10223) (2020) 507-513.

2. D. Wang, B. Hu, C. Hu, et al., Clinical characteristics of 138 hospitalized patients with 2019 novel coronavirus-infected pneumonia in Wuhan, China[J], JAMA 323 (11) (2020) 1061-1069, https://doi.org/10.1001/jama.2020.1585.

3. C. Huang, Y. Wang, X. Li, et al., Clinical features of patients infected with 2019 novel coronavirus in Wuhan, China[J], Lancet 395 (10223) (2020) 497-506.

4. Wu JT, Leung K, Leung GM. Nowcasting and forecasting the potential domestic and international spread of the 2019-nCoV outbreak originating in Wuhan, China: a modeling study. Lancet.2020. https://doi.org/10.1016/S01406736(20)30260-9.

5. Huang C, Wang Y, Li X, et al. Clinical features of patients infected with 2019 novel coronavirus in Wuhan, China. Lancet. 2020; S0140e6736(20): 30183e30185. https://doi.org/10.1016/ S0140-6736(20)30183-5.

6. Chen N, Zhou M, Dong X, et al. Epidemiological and clinical characteristics of 99 cases of 2019 novel coronavirus pneumonia in Wuhan, China: a descriptive study. Lancet. 2020; S0140e6736(20):30211e30217. https://doi.org/10.1016/ S01406736(20)30211-7.

7. Wang $\mathrm{D}, \mathrm{Hu} \mathrm{B}, \mathrm{Hu} \mathrm{C}$, et al. Clinical characteristics of 138 hospitalized patients with 2019 novel coronavirus-infected 
pneumonia in Wuhan, China. JAMA.2020. https://doi.org/10.1001/ jama.2020.1585.

8. Dong Y, Mo X, Hu Y, et al. (2020) Epidemiological Characteristics of 2143 Paediatric Patients With 2019 Coronavirus Disease in China. Pediatrics.

9. Moreton E (2020) Clinical management of severe acute respiratory infection (SARI) when COVID-19 disease is suspected. World Health Organisation.

10. Rodriguez-Morales AJ, Cardona-Ospina JA, Gutierrez-Ocampo E, Villamizar Pena R, Holguin-Rivera Y, Escalera-Antezana $\mathrm{JP}$, et al. Clinical, laboratory and imaging features of COVID-19: A systematic review and meta-analysis. Travel Med Infect Dis. (2020) 34:101623. doi: 10.1016/j.tmaid.2020.101623

11. Centers for Disease Control and Prevention. Resources for Emergency Health Professionals. Health Alert Netw 2020 https://emergency.cdc.gov/han/2020/ han00432.asp (accessed June 3.

12. Riphagen S, Gomez X, Gonzales-Martinez C, Wilkinson N, Theocharis P. Hyperinflammatory shock in children during COVID-19 pandemic Lancet2020; May 7. DOI:10.106/S0140-6736(20) 31094-1.

13. Verdoni L, Mazza A, Gerasoni A, et al. An outbreak of severe Kawasaki-like disease at the Italian epicentre of the SARS-CoV-2 epidemic: an observational cohort study. Lancet 2020 May 13. doi: 10.1016/S01406736(20)31103-X.

14. Hoang A, Chorath K, Moreira A, Evans M, Burmeister-Morton F, Burmeister F, Naqvi R, Petershack M, Moreira A. COVID-19 in 7780 pediatric patients: a systematic review. EClinicalMedicine. 2020 Jul 1;24:100433.
15. Rao S, Gavali V, Prabhu SS, Mathur R, Dabre LR, Prabhu SB, Bodhanwala M. Outcome of Children Admitted With SARSs-CoV-2 Infection: Experiences From a Pediatric Public Hospital. Indian pediatrics. 2021 Jan 11:S097475591600280.

16. Sarangi B, Reddy VS, Oswal JS, Malshe N, Patil A, Chakraborty M, Lalwani S. Epidemiological and clinical characteristics of COVID-19 in Indian children in the initial phase of the pandemic. Indian pediatrics. 2020 Oct;57(10):914-7. 